\title{
Preparation and quantitative magnetic studies of single-domain nickel cylinders
}

\author{
R. O'Barr, M. Lederman, ${ }^{\text {a) }}$ and S. Schultz \\ Department of Physics and Center for Magnetic Recording Research, University of California, \\ San Diego, 9500 Gilman Drive, La Jolla, California 92093-0319
}

Weihua $\mathrm{Xu}$ and $\mathrm{A}$. Scherer

Department of Electrical Engineering, California Institute of Technology, Pasadena, California 91125

\author{
R. J. Tonucci \\ Optical Sciences Division, Naval Research Laboratories, Washington DC 20375
}

\begin{abstract}
For a complete experimental and theoretical explanation of the magnetic processes in an interacting collection of submicron magnetic particles, a fundamental understanding of the magnetic properties of individual single-domain particles must first be achieved. We have prepared elongated $\mathrm{Ni}$ columns ranging in diameter from 0.15 to $1.0 \mu \mathrm{m}$ by electroplating into specially prepared $\mathrm{Al}_{2} \mathrm{O}_{3}$ and glass channeled pore membranes. We have also prepared controlled arrays of Ni columns using $e$-beam lithography, subsequently electroplating into the written patterns. Using transmission electron microscopy, we have characterized the shape, size, morphology, and crystal structure of the columns. Magnetic force microscopy has been used to determine the switching field $H_{s}$ versus the applied field angle of the columns. Although the switching field data can be fit to the functional form for nucleation by curling in an infinite cylinder, the observed weak dependence of $H_{s}$ on column diameter is inconsistent with that expected for curling, particularly for columns of diameter $>0.3$ $\mu \mathrm{m} . \quad$ (C) 1996 American Institute of Physics. [S0021-8979(96)40408-4]
\end{abstract}

\section{INTRODUCTION}

The magnetization state of a collection of magnetic particles is generally a complicated function of the applied field history and is a problem still lacking a complete theoretical description. The complexity is due mostly to the long range magnetostatic interactions between every pair of particles. Before a fundamental theory for the magnetization reversal of an interacting collection of particles can be formulated, one must first understand the reversal properties of the individual constituents, single-domain particles.

Our approach to this problem is to gather experimental data for the behavior of model single-domain particles specifically prepared for this purpose. Over the past few years, studies of the switching field versus applied field angle have been made on single-domain rectangular permalloy platelets, ${ }^{1}$ barium-ferrite platelets, ${ }^{2} \gamma-\mathrm{Fe}_{2} \mathrm{O}_{3}$ particles, ${ }^{3} \mathrm{Fe}$ particles, ${ }^{4}$ rectangular Ni platelets, ${ }^{5}$ and long $\mathrm{Ni}$ cylinders. ${ }^{6}$ In this article, we describe the preparation, characterization, and magnetic measurements of single-domain elongated $\mathrm{Ni}$ columns, and a method used to prepare controlled assemblies of Ni columns in designed configurations.

\section{SAMPLE PREPARATION}

The method we have used to prepare samples consists of electroplating $\mathrm{Ni}$ into channel pore membranes prepared by three different techniques:

\section{A. Anodized aluminum}

One type of porous membrane which we have filled by electroplating are $\mathrm{Al}_{2} \mathrm{O}_{3}$ filters commercially available from Whatman Inc. ${ }^{7}$ Details of the set up and the plating condi-

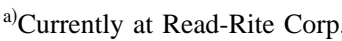

tions used have been previously reported. ${ }^{6}$ The standard Whatman $\mathrm{Al}_{2} \mathrm{O}_{3}$ filters have diameters ranging from $\sim 0.25-$ $0.45 \mu \mathrm{m}$ and we can vary the length of the columns prepared by changing the duration or the current density used in the plating process. We have also utilized a series of custom made filters for which the anodization conditions have been adjusted to produce pores with smaller hole sizes.

\section{B. Nanochannel glass arrays}

A second type of porous membranes are nanochannel glass arrays developed by Tonucci et al., ${ }^{8}$ with pore diameters of $1 \mu \mathrm{m}$. Nanochannel glass membranes contain highly uniform parallel pores or channels in large arrays. Samples are prepared by polishing a channel glass wafer to a thickness appropriate for the desired column length. The wafer is then etched with a weak acetic acid solution exposing the channels in the array. The channel glass wafer is then sealed over a hole in a glass cover slip in preparation for electrodeposition. Details of the plating conditions were similar to that used for the anodized aluminum samples discussed above.

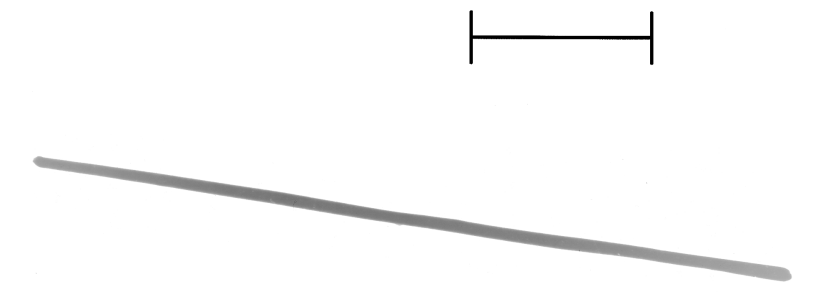

FIG. 1. Bright field TEM image of column $\alpha$ which has an aspect ratio of $63: 1$...Scale bar is $4 \mu \mathrm{m}$. 


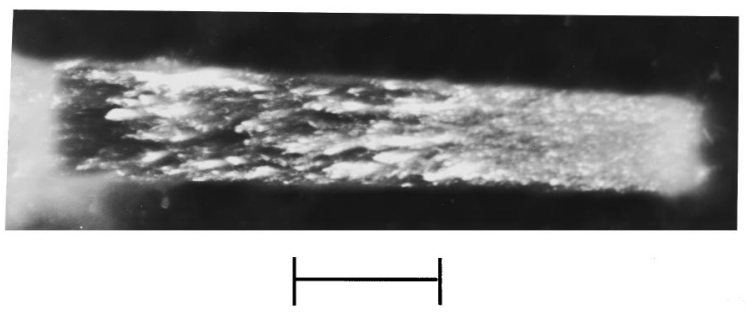

FIG. 2. Dark field TEM image of a particle written by $e$-beam lithography. The scale bar is $0.2 \mu \mathrm{m}$.

\section{Electron-beam lithography}

A third method for preparing columnar pores utilizes electron-beam lithography as reported by Chou et al. ${ }^{9}$ The details of our lithography will be included in Ref. 10. In brief, a thin gold layer is evaporated onto a semiconducting or insulating substrate in a pattern that consists of a beamwriting pad and two electrically connected probe contact pads. We then spin-coat a single, high molecular weight polymethyl methacrylate (950k PMMA) resist layer. The resist is then patterned using a Phillips EM-430 scanning transmission electron microscope (STEM) with an acceleration voltage of $250 \mathrm{kV}$. Following the lithographic exposure, the exposed patterns are developed in a 3:7 cellulose-methanol mixture. Electrodeposition at constant current is then carried out using a nickel sulfamate electrolyte. Using this technique, we have prepared columns ranging from 0.02 to 0.2 $\mu \mathrm{m}$ in diameter, and exceeding $1 \mu \mathrm{m}$ in length.

\section{SAMPLE CHARACTERIZATION}

To determine the size, shape, morphology, and crystal structure of the Ni columns, we first remove the surrounding membrane. The $\mathrm{Al}_{2} \mathrm{O}_{3}$ filters and nanochannel glass membranes are removed by etching in $1 \mathrm{M} \mathrm{NaOH}$. The PMMA is removed by an oxygen plasma etch. The columns can then be observed directly in a SEM or placed in water, ultrasonicated, and evaporated onto a transmission electron microscopic (TEM) grid. Figure 1 shows a bright field image of a

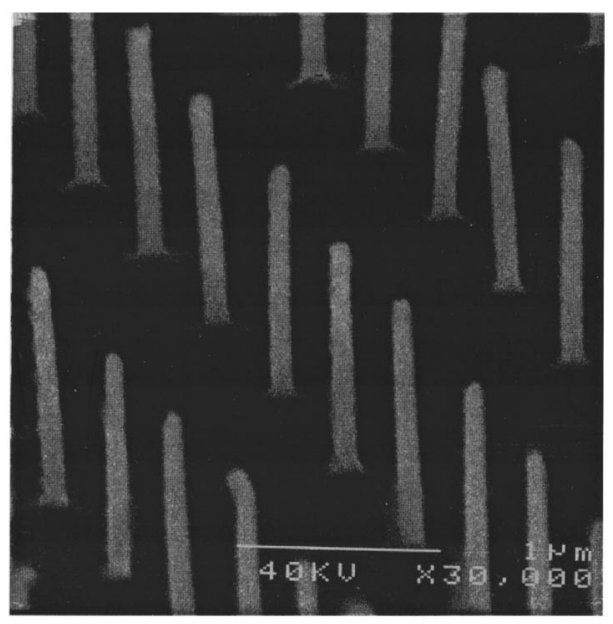

FIG. 3. SEM micrograph of an array of columns written by $e$-beam lithography after removal of the PMMA layer. Column height is $>1 \mu \mathrm{m}, d=0.1$ $\mu \mathrm{m}$, and the spacing between columns $\sim 1 \mu \mathrm{m}$.

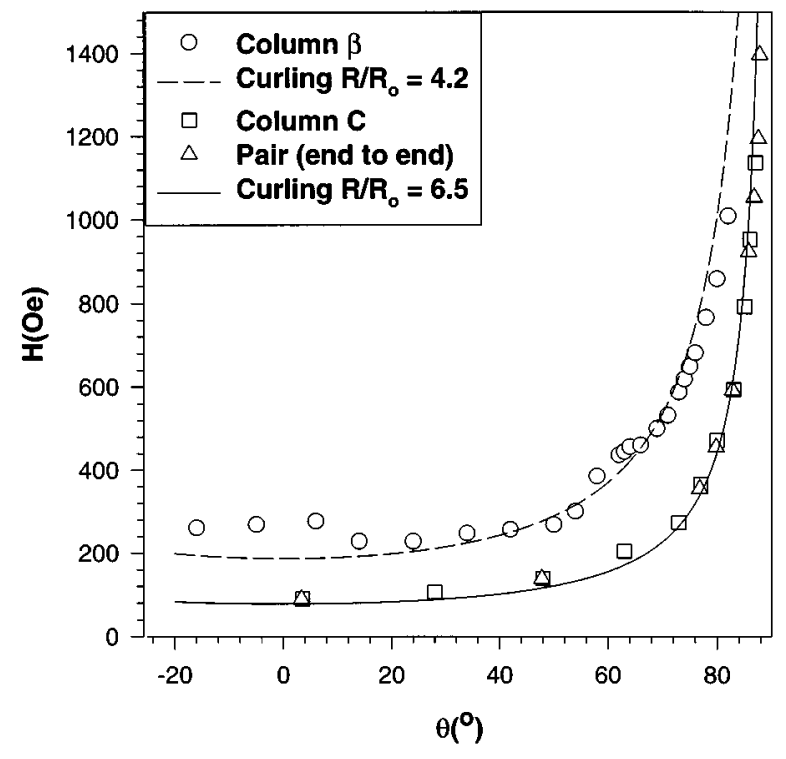

FIG. 4. Switching field vs angle between external field and the long axis, $H_{s}(\theta)$ of column $C$, and a pair of similar columns aligned end-to-end $(d=1$ $\mu \mathrm{m}$, and $L=44 \mu \mathrm{m})$, and for column $\beta(d=.15 \mu \mathrm{m}, L=1.65 \mu \mathrm{m})$. The solid and dashed curves correspond to $H_{s}(\theta)$ for nucleation by curling in an infinite cylinder. For $\mathrm{Ni}, R_{0}=\sqrt{ } A / M_{S}$ is $206 \AA$, where $A$ is the exchange constant, and $M_{s}$ is the saturation magnetization.

column previously designated as $\alpha,{ }^{6}$ prepared using an $\mathrm{Al}_{2} \mathrm{O}_{3}$ filter. This column has a diameter $d=0.27 \mu \mathrm{m}$, length $L=17$ $\mu \mathrm{m}$, and an aspect ratio of 63:1.

Figure 2 is a dark field image of a particle written by $e$-beam lithography. As can be seen in this image, the crystal structure is polycrystalline with a decrease in the grain size as the plating progresses. We intend to improve the crystallinity by varying and optimizing the plating parameters. An illustration of an array of columns written by $e$-beam lithography as visualized by SEM after the removal of the PMMA, is shown in Fig. 3.

\section{MEASUREMENT OF MAGNETIC PROPERTIES}

Using a magnetic force microscope $(\mathrm{MFM})^{11}$ with an in situ electromagnet, we have measured the switching field versus applied field angle for several different columns. Columns are first located and characterized by TEM on a grid, and then relocated by correlation of various marking features in the MFM. The measurement sequence is as follows. A reverse field of 1650 Oe is pulsed in a direction making an angle $\theta$ with the long axis of the column for $1 \mathrm{~s}$. This field is verified to be sufficient to reverse saturate the column's magnetization. A forward field $H$, is then applied for $2 \mathrm{~s}$. Before the fields are applied, the MFM tip is backed away to a distance of $12 \mu \mathrm{m}$. After the application of the field pulses, the column is then imaged in the remanent state to see if the forward field $H$ was sufficient to reverse the direction of magnetization. The lowest $H$ necessary to switch the direction of the magnetization of the column is defined as the switching field $H_{s}$ for that angle.

The results for an isolated column, prepared with nanochannel glass membranes (designated as $C$ ) and for two 


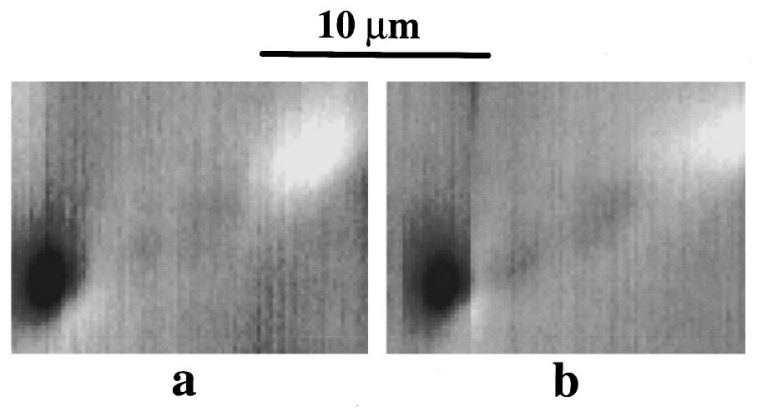

FIG. 5. MFM images of column $A(d=.45 \mu \mathrm{m}, L=12.7 \mu \mathrm{m})$ scanned in remanence after applying a field of (a) $H=0$ and (b) $H=475$ Oe. For this particular case, $H_{s}=480$ Oe.

similar columns aligned end-to-end, each with $d=1 \mu \mathrm{m}$ and $L=44 \mu \mathrm{m}$, and for an isolated column prepared with an $\mathrm{Al}_{2} \mathrm{O}_{3}$ filter (designated $\beta$ ) are presented in Fig. 4. The solid and dashed curves correspond to the analytical micromagnetic solution of $H_{s}(\theta)$ for curling ${ }^{12}$ in an infinite cylinder where we have adjusted $R / R_{0}$ to get a best fit, where $R$ is the cylinder radius and $R_{0}$ is the critical radius for curling. Even though we don't expect curling to be valid over the entire range, in Fig. 4 we plot the relationships for comparison. MFM images, such as those in Fig. 5, show that magnetic charge is concentrated only at the ends of the columns, with negative charge at one end and positive at the other, with the polarity (of the magnetization) dependent on whether the previously applied field $H>H_{s}$. At remanence, a multidomain state has never been observed, even if the previously applied field was near $H_{s}$ (see Fig. 5). It is surprising that even the columns with $d=1 \mu \mathrm{m}$ are single-domain which may be due to the large aspect ratio which should help to stabilize the single-domain state. The angular dependence for column $C$ is similar to that of columns $1,2(d=0.3 \mu \mathrm{m}$, $L=5.5 \mu \mathrm{m}), A, B(d=0.45 \mu \mathrm{m}, L=12.7 \mu \mathrm{m})$, and $\alpha(d=0.27$ $\mu \mathrm{m}, L=17 \mu \mathrm{m})$ given in Fig. 2 of Ref. 6 . The angular dependence for column $\beta(d=0.15 \mu \mathrm{m}, L=1.65 \mu \mathrm{m})$ is different in that $H_{s}$ increases slightly as $\theta$ approaches $0^{0}$. The switching field at zero degrees $H_{s}\left(\theta=0^{0}\right)$ versus column radius $R$ is plotted in Fig. 6 . The solid line is the solution for the nucleation field via curling in an infinite $\mathrm{Ni}$ cylinder. Column $\beta$ has a zero angle switching field of $276 \mathrm{Oe}$, a value close to the theoretical curve.

\section{CONCLUSION}

We have prepared columns by electroplating into porous membranes ranging in diameter from 0.15 to $1.0 \mu \mathrm{m}$ and have measured the switching field versus applied field angle. We have also demonstrated the capability to prepare arrays of columns with diameters as small as.02 $\mu \mathrm{m}$ using $e$-beam lithography. In general, the observed angular dependence of the switching field (Fig. 4) agrees with the functional form

\section{$\mathbf{H}_{\mathbf{s}}\left(\theta=0^{\circ}\right)$ vs. radius $(\mu \mathrm{m})$}

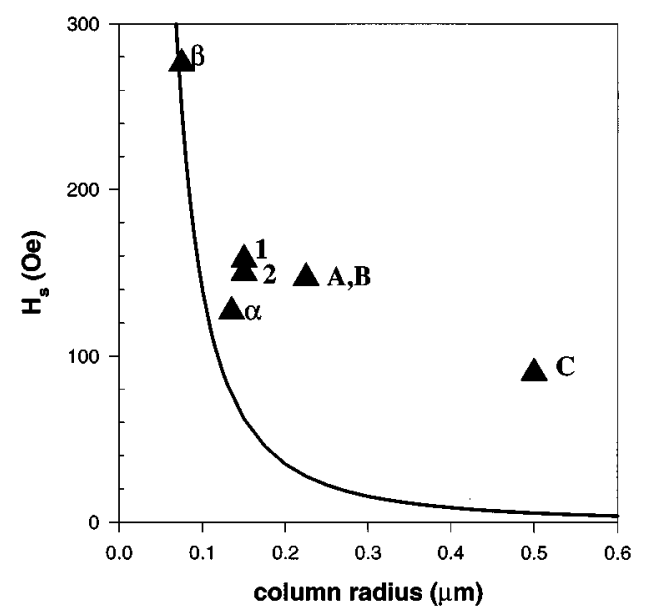

FIG. 6. Switching field when the external field is aligned with the column long axis vs column radius. Solid line is the solution given for nucleation by curling in an infinite cylinder.

corresponding to curling in an infinite cylinder, although at the expense of reassigning the $R / R_{0}$ value to get a best fit. For Fig. 4 that adjustment is from 24.3 to 6.5 for column $C$ and from 3.64 to 4.2 for column $\beta$. For $d>0.3 \mu \mathrm{m}$, the dependence of $H_{s}$ on diameter is weak which is not consistent with curling and may indicate another mode for initiating reversal such as nucleation at the particle ends.

\section{ACKNOWLEDGMENTS}

Work was sponsored by the Center for Magnetic Recording Research, NSF Grant No. DMR-94-00439 (MRSEC), and ARPA/ETP.

${ }^{1}$ M. Lederman, G. A. Gibson, and S. Schultz, J. Appl. Phys. 73, 6961 (1993).

${ }^{2}$ T. Chang and J. Zhu, J. Appl. Phys. 75, 5553 (1994).

${ }^{3}$ M. Lederman, D. R. Fredkin, R. O'Barr, S. Schultz, and M. Ozaki, J. Appl. Phys., 75, 6217 (1994); C. Salling, R. O’Barr, S. Schultz, I. McFadyen, and M. Ozaki, ibid. 75, 7989 (1994).

${ }^{4}$ Y. Luo and J. Zhu, IEEE Trans. Magn. 30, 4080 (1994).

${ }^{5}$ S. Chou, P. Krauss, W. Wei, and P. Fischer, Scr. Metall. Mater. 33, 1537 (1995).

${ }^{6}$ M. Lederman, R. O’Barr, and S. Schultz, IEEE Trans. Magn. 32, 3793 (1995).

${ }^{7}$ Whatman Corp. Catalog Number 20046S50.

${ }^{8}$ R. J. Tonucci, B. L. Justus, A. J. Campillo, and C. E. Ford, Science 258, 783 (1992).

${ }^{9}$ S. Y. Chou, M. S. Wei, P. R. Krauss, and P. B. Fischer, J. Appl. Phys. 76, 6673 (1994).

${ }^{10}$ H. Xu, J. Wong, C. C. Cheng, R. Johnson, and A. Scherer, J. Vac. Sci. Technol. B 13, 2372 (1995).

${ }^{11}$ G. A. Gibson, J. F. Smyth, S. Schultz, and D. P. Kern, IEEE Trans. Magn. 27, 5187 (1991).

${ }^{12}$ E. H. Frei, S. Shtrikman, and D. Treves, Phys. Rev. 106, 446 (1957); S. Shtrikman and D. Treves, J. Physique (Paris) 20, 286 (1959). 